\title{
How I do it
}

Bertram Ng, MD Hong Kong, China ngbertram@yahoo.com.hk

Dr. James Harris needs no further introduction. He has been devoted to refining the art of FUE, and has introduced the concept of blunt dissection. Here he shares with us pearls for performing powered FUE. Dr. Harris emphasizes the importance of magnification. My personal experience is the very narrow visual field associated with anything higher than $3.5 \times$. For those working alone, the constant shift of visual fields when looking for instruments creates considerable eye strain.

\section{Powered blunt dissection with the SAFE System for FUE (Part I)}

\section{James A. Harris, MD Greenwood Village, Colorado jaharris@hsccolorado.com}

Disclosure: The author has ownership interest in HSC Development, LLC, which manufactures and sells the Powered SAFE System for FUE. Since no claims are made in this article, there is no real conflict of interest as it is instructional in nature.

Even though technology has provided tools to enhance the speed, efficiency, and accuracy of FUE procedures, there are still definite steps and details that need to be followed in order to make the most of the equipment.

The Power SAFE System (HSC Development, Greenwood Village, $\mathrm{CO}$ ) uses only a blunt punch. It is "one step" with no other steps for dissection. My experience with this system over the past three years and hundreds of thousands of grafts has resulted in relatively straightforward, but critical, steps to follow.

\section{Preparation Steps}

Before wielding the instrument, there are three critical things the physician must do to ensure that the remainder of the case will go smoothly:

1. Use adequate magnification and lighting. If you can't see your target, you won't ever be "on target." The number one reason that a physician can't perform FUE is that he or she can't get the target unit lined up properly in the lumen of the dissecting punch. I strongly suggest that a physician use $6 \times$ surgical loupes, if not, then a minimum of $5 \times$. Any lighting that allows the physician to see the hair should be adequate.

2. Trim the hair to $1.5-2.0 \mathrm{~mm}$; this will aid in a speedy positioning of the hair in the punch lumen. If the hair is too long, the doctor will spend too much time "threading" the hair into the punch. I find that the use of a Wahl "Peanut" beard trimmer will trim the hair to the proper length.

3. Position the patient and yourself so that all hand and arm motion is towards you; that is, you should be standing (or sitting) in a position as close as possible opposite the direction of hair growth.

If you can master the above steps, you are well on your way to successful power FUE. As easy as these may seem, you can't imagine the reluctance of physicians to use adequate magnification, insisting that they see fine with $2-3 \times$ loupes. I can assure you that you can't.

\section{Extraction Steps}

The next steps involve the preparation of the equipment, the area to be harvested, and the proper use of the equipment:

1. Donor area injection. I will inject the donor area, into the fat, with either plain saline or a 1:100,000 epinephrine solution in saline. I typically use about $0.5 \mathrm{cc} / \mathrm{cm}^{2}$ and only inject the area that can be harvested in about 10-15 minutes. This is not a "tense" tumescence as you will only see a slight elevation of the skin. This process will help with hemostasis and provide some subcutaneous pressure to elevate the dissected grafts. This injection is in addition to the ring block for anesthesia.

2. Speed control adjustment. There is an initial setting, as suggested in the user instructions, in which the indicator is placed in the first third of the speed range. The critical step, however, is the adjustment of the speed based on the initial extraction attempts on the patient. The speed has to be fast enough to allow an easy entry of the punch into the skin, and once the punch is advanced, the rotation speed slows significantly. When a physician is learning the procedure, I would suggest that it slows enough that the individual rotations can be discerned by observation. The physician should also hear the rotation speed diminish. Graft dissection in different parts of the scalp may require changes in speed settings.

3. Skin traction. Traction should be placed on the skin opposite to the direction of hair emergence. The amount of traction placed should eliminate skin motion when the punch is placed on the skin in the next step.

4. Punch placement and engagement. Using adequate magnification, the punch should be aligned with the emerging hair shafts and at the approximate angle of emergence. With the skin under traction, the punch rotating, the tip should be placed over the hair and pressed into the skin so that a definite indentation into the skin should be noted; this is the engagement (Figure 1). After a brief pause, the punch should be advanced slowly into the skin to the depth limiter and the doctor should listen and observe for a slowing of the rotation speed; if none is noted, the speed should be decreased. 
5. Evaluation. If there was a proper dissection, the graft will usually elevate $0.5-1 \mathrm{~mm}$ from the skin surface (Figure 2). This should be monitored for every dissection. If a graft is buried or not elevating, the usual cause is that the insertion angle was not acute enough and the corrective response is to drop the angle (move the punch closer to the skin) by 4-5 degrees. I will typically dissect 5-10 grafts at the beginning of the case to ensure angles, directions, and speed settings are correct.

If transections are noted, the incomplete graft is removed and analyzed. Most of the time, transections are caused by an entry angle that is not acute enough, however, occasionally, it may be due to an angle that is too acute. $\vec{\gamma}$

To be continued in the next installment of How I Do It.

Editorial note: The pearls described in this column are obviously applicable to the particular instrument the author developed and utilizes, and other powered follicular unit extraction instruments may have subtle but significant differences in their use. With the goal of keeping our members abreast of all developments in FUE instrumentation, the Editors take this opportunity to welcome users of different powered or manual FUE instruments to submit their personal experiences to share with our readers, as Dr. Harris does in this How I Do It section.

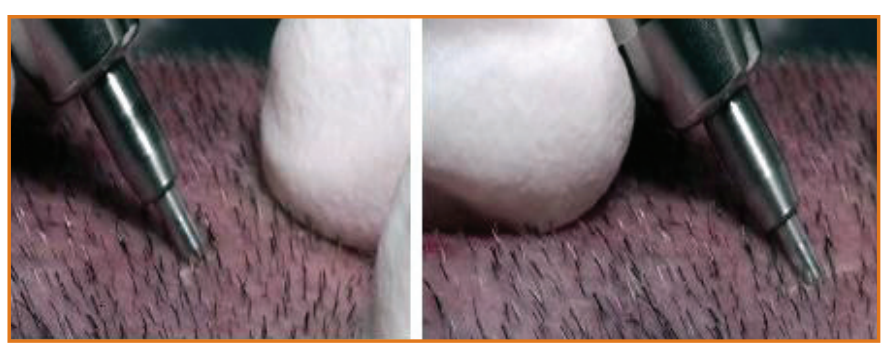

Figure 1. The subtle depression of the skin during punch "engagement" is seen in the photo on the right. This is performed before advancing the punch.

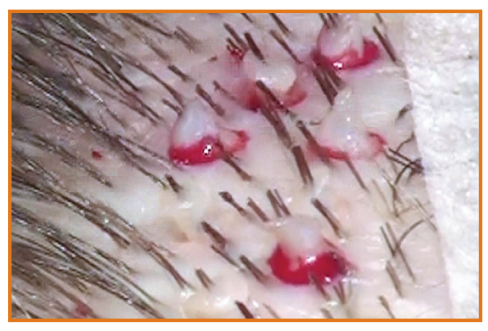

Figure 2. Properly dissected grafts will usually elevate slightly.

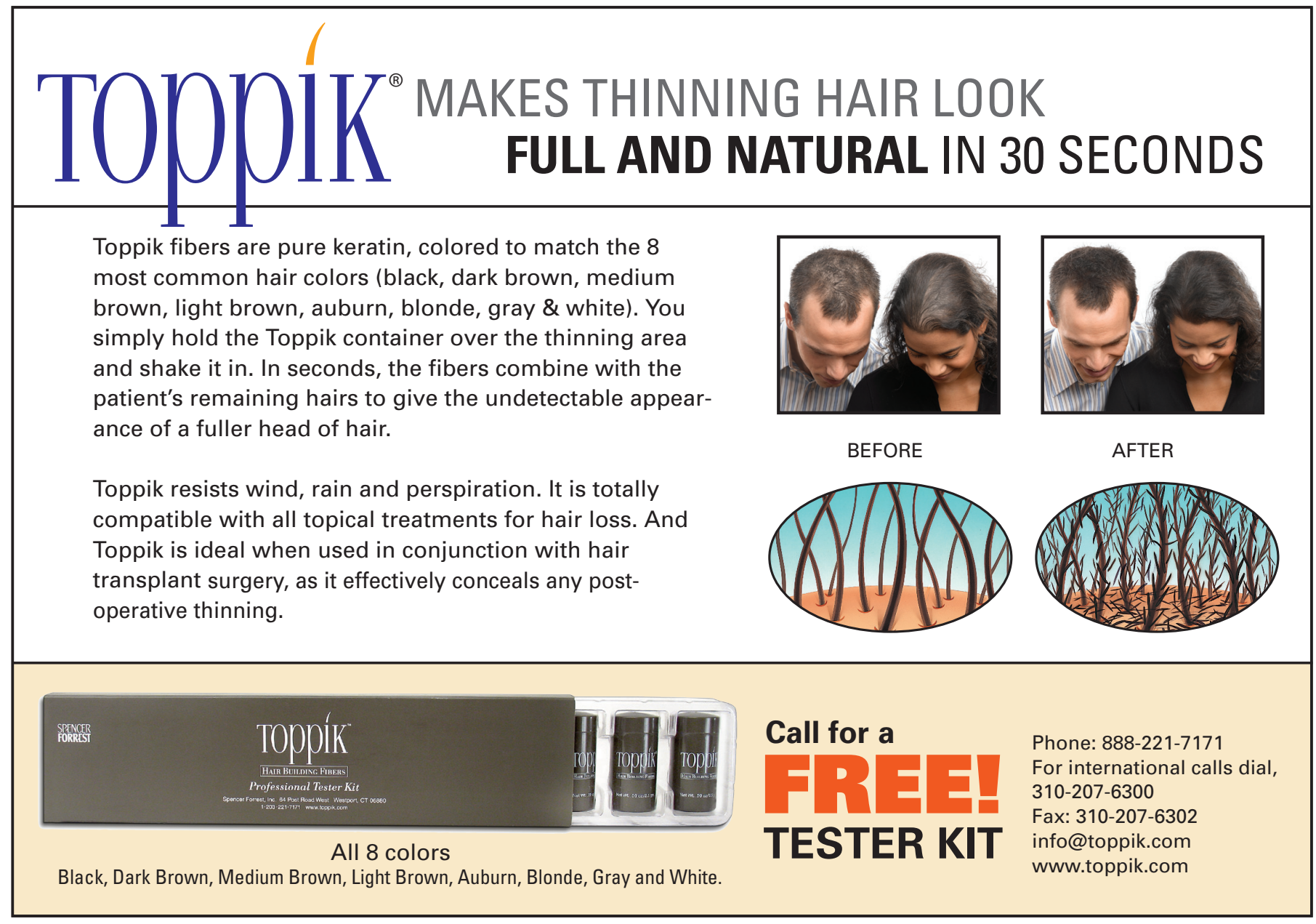

